\title{
Study on biological distribution of polyhexamethylene guanidine (PHMG), a toxic household chemical, using radiolabeling and molecular imaging tools
}

\author{
Sajid Mushtaq ${ }^{12^{*}}$, Jung Eun Park ${ }^{3^{*}}$, Ha Eun Shim ${ }^{2}$, Chang Heon Lee ${ }^{2}$, Hee Soon Shin ${ }^{4,5}$, \\ So-Young Lee ${ }^{4,5}$, Jongho Jeon ${ }^{3+}$ \\ ${ }^{1}$ Department of Nuclear Engineering, Pakistan Institute of Engineering and Applied Sciences, Islamabad 45650, Pakistan \\ ${ }^{2}$ Advanced Radiation Technology Institute, Korea Atomic Energy Research Institute, Jeongeup 56212, Republic of Korea \\ ${ }^{3}$ Department of Applied Chemistry, College of Engineering, Kyungpook National University, Daegu 41566, Republic of Korea \\ ${ }^{4}$ Division of Functional Food Research, Korea Food Research Institute, Wanju-gun, Jeollabuk-do 55365, Republic of Korea \\ ${ }^{5}$ Food Biotechnology Program, University of Science and Technology, Daejeon 34113, Republic of Korea \\ *These authors contributed equally to this work.
}

\begin{abstract}
A lot of people in Korea have suffered from severe toxicity of PHMG, a humidifier disinfectant. To assess the risk and in-depth mechanistic understanding of toxicity posed by a hazardous substance, it is essential to determine its biological uptake in organs. In this study, we investigate the biodistribution of PHMG using radioanalytical techniques. By labeling PHMG with radioactive indium ( ${ }^{111}$ In), the uptake values of PHMG in organs can be measured after intravenous injection in Sprague-Dawley rats for up to $168 \mathrm{~h}$. The results indicate that PHMG is distributed in the liver $(28.63 \%$ injected dose (\%ID)) and lungs $(12.44 \% \mathrm{ID})$ at $30 \mathrm{~min}$ post administration. In addition, the clearance rate of PHMG distributed in the internal organs is slow, with approximately 65\% of injected PHMG remaining in the lungs after 1 week. Moreover, we quantified biodistribution of the radiolabeled PHMG exposed through intratracheal instillation and oral administration routes, and the observed data were compared to each other to understand in vivo behavior of PHMG. In addition, radiolabeled PHMG in an animal model can be visualized using SPECT/CT. The obtained results indicate that repetitive exposure to PHMG results in damage to lungs, as well as extrapulmonary organs.
\end{abstract}

Keywords: Biodistribution, Household disinfectant, Molecular imaging, Polyhexamethylene guanidine, Radiolabeling

\section{Introduction}

Polyhexamethylene guanidine (PHMG) is a positive charged oligomeric and polymeric compound that has been applied for many years in several industries [1, 2]. It contains multiple guanidine groups and is known as a potent biocide with a broad spectrum of bioactivities [3, 4]. When PHMG was produced as a household product, its toxicity to the human body was underestimated due to poor or wrong evaluation experiments, making many people in Korea suffer from a tragic outbreak of severe pulmonary disorders. The lung diseases originated from the use of PHMG as a humidifier disinfectant [5-8]. For more than 10 years, approximately 200 people have died, among them children and pregnant women as well, from the inhalational toxicity of PHMG. Moreover, according to the press release reported by the Ministry of Environment, several hundred people continue to have serious respiratory diseases caused by the humidifier disinfectant [9]. A lot of studies have confirmed that the inhalation of PHMG caused lung injuries, such as acute inflammation and catastrophic fibrogenesis [10-13]. In addition, various studies have been conducted or are under investigation to verify the toxicity of PHMG to other internal organs of the human body [14-18].

To evaluate organ toxicity on exposure to toxic substances, its uptake, distribution, and excretion profile need to be determined

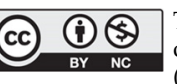

This is an Open Access article distributed under the terms of the Creative Commons Attribution Non-Commercial License (http://creativecommons.org/licenses/by-nc/3.0/) which permits unrestricted non-commercial use, distribution, and reproduction in any medium, provided the original work is properly cited.

Copyright (C) 2022 Korean Society of Environmental Engineers
Received August 20, 2021 Accepted October 06, 2021

${ }^{\dagger}$ Corresponding author

E-mail: jeonj@knu.ac.kr

Tel: +82-53-950-5584

ORCID: 0000-0002-0288-0991 


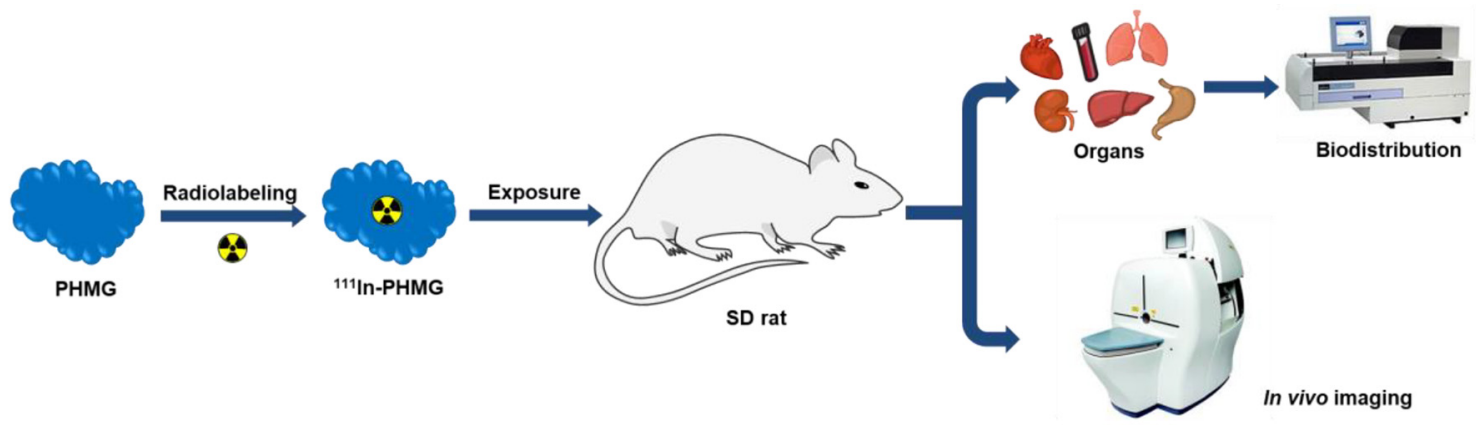

Fig. 1. Study design for biodistribution and single-photon emission computed tomography imaging study based on ${ }^{111}$ In-labeled polyhexamethylene guanidine.; SD, Sprague-Dawley.

in a living subject $[19,20]$. However, the measurement of the biological uptake of PHMG is difficult due to the limited detection techniques in tissues, which contain complex chemical species and biomolecules. Mass spectrometry cannot be used to analyze PHMG in biological samples since PHMG comprises a mixture of polymers with many molecular weights. In addition, this material does not generate a convenient optical signal nor adsorption spectrum, and thus ultraviolet-visible and fluorescent detectors would not be useful to detect trace quantities in vivo. These limitations can be overcome using a radionuclide as a tracer. The radiation energy (i.e., $\gamma$-ray) emitted from a conventionally used radionuclide in nuclear medicine can penetrate biological tissues without any signal interference. Therefore, the method employing a radiotracer allows straightforward, accurate, and sensitive detection, as well as non-invasive imaging in a living subject [21-25]. In the previous study, we have reported the radiolabeling of PHMG to determine uptake values in animal models [26]. The radioactive indium $\left({ }^{111} \mathrm{In}\right)$-labeled PHMG aerosol was exposed to SpragueDawley (SD) rats by inhalation, following which the toxic chemical in biological tissues was measured. The high accumulation and retention of PHMG aerosols in the lungs, as well as transport from the lungs, were observed quantitatively through the experiment. Although these results suggest the severe inhalation toxicity of PHMG, further in vivo assessment is still necessary for an in-depth understanding of its effect in other internal organs. In this study, we report the biodistribution of radiolabeled PHMG following intravenous injection for 1 week to investigate organ-specific uptake, retention, and clearance of PHMG. Then, the results will be discussed by comparing other organ distribution results obtained by the intratracheal instillation and oral administration, which are considered main exposure routes of PHMG. In addition, non-invasive whole-body images are provided to visualize PHMG administered in animal models. Fig. 1 illustrates the study design, which includes the organ distribution study and single-photon emission computed tomography (SPECT) imaging using the radiolabeled PHMG.

\section{Materials and Methods}

\subsection{General Procedure}

The radioisotope $\left(\left[{ }^{111} \mathrm{In}\right] \mathrm{InCl}_{3}\right)$ was supplied by Mallinckrodt
Pharmaceuticals (Netherlands). The PHMG solution (25 wt\% in water, SKYBIO 1125, weight average molecular weight $=$ ca. $4.1 \mathrm{kDa}$ ) was provided by the Korea Institute of Toxicology (Jeongeup, Republic of Korea). All commercially available reagents were used without further purification. For the preparative high-performance liquid chromatography (HPLC) experiment, $0.1 \%$ formic acid containing $\mathrm{H}_{2} \mathrm{O}$ (solvent $\mathrm{A}$ ), and $0.1 \%$ formic acid, containing acetonitrile (solvent $\mathrm{B}$ ), were used as eluents (Agilent 1260 Infinity, USA). The flow rate was $10 \mathrm{~mL} / \mathrm{min}$ for preparative HPLC (column: Eclipse XDB-C18, $21.2 \times 150 \mathrm{~mm}$, $7 \mathrm{~mm}$, eluent gradient: $100 \%$ solvent A for 0-5 min; $30 \%$ solvent $\mathrm{B}$ in solvent A for 5-20 min; 70\% solvent B in solvent A for 20-25 min; $100 \%$ solvent A for $25-26 \mathrm{~min}$; retention time of PHMG-DOTA: 20.0-24.5 min). The amount of radioactivity was measured using $\gamma$-counter (1480 Wizard 3, PerkinElmer, USA). The radiolabeling reaction was monitored using a radio-thin-layer chromatography (radio-TLC) imaging scanner (AR-2000, Eckert \& Ziegler, USA). The SPECT images were obtained using a small animal SPECT/PET/CT system (Siemens, USA).

\subsection{Preparation of Polyhexamethylene Guanidine (PHMG)- DOTA and Radiolabeling of PHMG}

The conjugation of PHMG with DOTA (1,4,7,10-tetraazacyclodecane-1,4,7,10-tetraacetic acid) and ${ }^{111}$ In labeling was conducted following the methods in our previous report [26]. Aqueous PHMG (25 wt\%, SKYBIO 1125) reacted with three equivalent of $p$-SCN-Bn-DOTA for $6 \mathrm{~h}$ at $37^{\circ} \mathrm{C}(\mathrm{pH}=8)$. Unreacted or hydrolyzed DOTA in the crude mixture was removed using preparative HPLC purification. The product was characterized using nuclear magnetic resonance spectroscopy. PHMG-DOTA conjugate was radiolabeled by adding $148 \mathrm{MBq}$ of [ $\left.{ }^{111} \mathrm{In}\right] \mathrm{InCl}_{3}$ in $0.1 \mathrm{M}$ aqueous $\mathrm{HCl}$ to 1 mg of PHMG-DOTA in $50 \mu \mathrm{L}$ pure water. The $\mathrm{pH}$ was adjusted to 4 using aqueous sodium hydroxide, and the resulting mixture was heated at $80^{\circ} \mathrm{C}$ for $15 \mathrm{~min}$ and then cooled to $25^{\circ} \mathrm{C}$. Radiolabeling was monitored using radio-TLC, as shown in Fig. 2(b). The radiochemical purity of the final product $\left({ }^{111} \mathrm{In}-\mathrm{PHMG}\right)$ was determined using radio-TLC.

\subsection{Animal Care for in Vivo Experiments}

Male SD rats (aged 8 weeks, 250-270 g) obtained from Orient Bio Inc. (Iksan, Republic of Korea) were used for in vivo imaging and biodistribution experiments. Animals were randomly divided into 
seven groups (five rats per group) for biodistribution studies. They were kept in ventilated cages under standard conditions of temperature, humidity, pressure, and light (light/dark cycle for $12 \mathrm{~h}$ ). Animals were provided with a sterile diet and water ad libitum. The protocols of animal studies were approved by the Institutional Animal Ethical Committee (Korea Atomic Energy Research Institute) and performed under strict compliance with the guidelines issued by the committee.

\subsection{Biodistribution Study of ${ }^{111}$ In-labeled Polyhexamethylene Guanidine}

${ }^{111} \mathrm{In}$-PHMG (37 KBq/100 $\left.\mu \mathrm{L}\right)$ in saline was administered to SD rat body (weight: 250-270 g) through different administration routes (intravenous injection, intratracheal instillation, oral administration). At each time point $(0.5,3,6,18,48,72$, and 168 h), five rats were sacrificed under anesthesia and the organs (heart, lungs, liver, spleen, kidneys, stomach, small intestine, large intestine, and thyroid) and the blood were harvested. The radioactivity of the collected organs was measured using $\gamma$ -counter. The distribution data were shown as percentages of injected dose (\%ID) or percent of injected dose per gram of tissue (\%ID/g).

\subsection{Single-photon Emission Computed Tomography/Computed Tomography Imaging of ${ }^{111}$ In-labeled Polyhexamethylene Guanidine}

The animal was anesthetized with $2 \%$ isoflurane. ${ }^{111} \mathrm{In}$-PHMG (5.6 $\mathrm{MBq} / 100 \mu \mathrm{L}$ ) solution was administered through different administration routes (intravenous injection and intratracheal installation). SPECT/computed tomography (CT) images were obtained using a small animal SPECT/CT system (Siemens, USA) at $0.5,1.5$, and $18 \mathrm{~h}$ (or $24 \mathrm{~h}$ ) post administration.

\section{Results and Discussion}

\subsection{Preparation of Radiolabeled Polyhexamethylene Guanidine (PHMG) (111 In-PHMG)}

To introduce ${ }^{111}$ In to the PHMG molecule, the amino group of PHMG was first conjugated with DOTA (Fig. 2(a)). For efficient incorporation of a metal chelating agent, three equivalents of DOTA-isothiocyanate were mixed with PHMG solution for $6 \mathrm{~h}$ at $\mathrm{pH}=8$ and $37^{\circ} \mathrm{C}$. At the end of the reaction, the crude product was purified, using a preparative HPLC, to give DOTA conjugated PHMG (PHMG-DOTA). The radiolabeling was performed by mixing an aqueous solution of PHMG-DOTA with [ $\left.{ }^{111} \mathrm{In}\right] \mathrm{InCl}_{3}$ for $15 \mathrm{~min}$ at $\mathrm{pH}=4$ and $80^{\circ} \mathrm{C}$. Radio-TLC analysis showed that the $\mathrm{R}_{\mathrm{f}}$ value of free ${ }^{111}$ In metal was 0.55 , whereas that of radiolabeled PHMG ( $\left.{ }^{111} \mathrm{In}-\mathrm{PHMG}\right)$ was 0.29 when aqueous citric acid $(0.1 \mathrm{M})$ was used as an eluent (Fig. 2(b)). The radiolabeling was accomplished with a high radiochemical yield and the amount of unbounded radioactive indium $\left(\left[{ }^{111} \mathrm{In}\right] \mathrm{InCl}_{3}\right)$ in the reaction mixture was negligible. As the radiolabeling procedure was straightforward and provided high radiochemical purity ( $>$ 99\%), analyzed by radio-TLC, no further purification was necessary for the next experiments.

\subsection{In vitro Stability Studies}

The stability of the radiolabeled PHMG was tested in mouse serum at $37^{\circ} \mathrm{C}$. In this study, $10 \mu \mathrm{L}(3.7 \mathrm{MBq})$ of the desired ${ }^{111} \mathrm{In}-\mathrm{PHMG}$ was incubated with $90 \mu \mathrm{L}$ serum in a microcentrifuge tube $(0.6$ $\mathrm{mL}$ ) at the abovementioned temperature. The amount of intact ${ }^{111}$ In-PHMG was determined using radio-TLC at 0.5, 3, 6, 18, 48, 72, and $168 \mathrm{~h}$. However, the results of the radio-TLC chromatogram were confusing, as the metabolized or protein-bounded radiotracer showed broad $R_{\mathrm{f}}$ values, which impede accurate integration for analyzing the stability in the serum. Thus, radio-HPLC was used
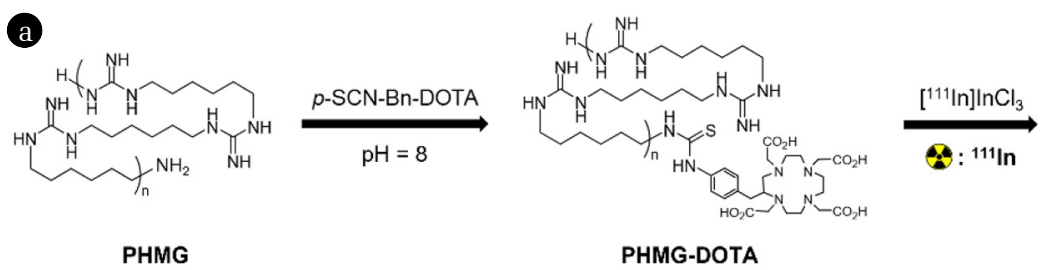

PHMG-DOTA
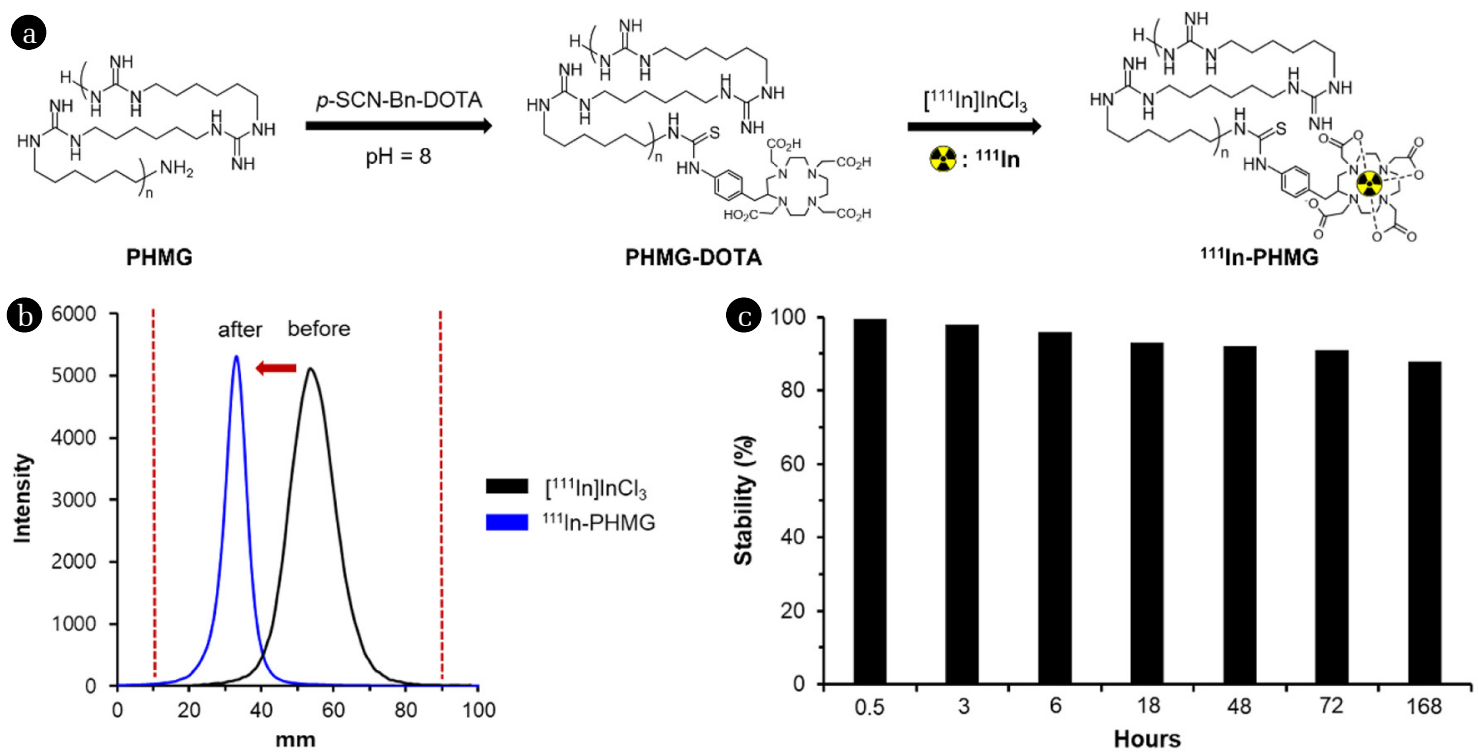

Fig. 2. (a) Radiolabeling procedure for preparation of ${ }^{111}$ In-polyhexamethylene guanidine (PHMG). (b) Radio-thin-layer chromatogram of $\left[{ }^{111} \mathrm{In}\right] \mathrm{InCl} \mathrm{I}_{3}$ and ${ }^{111} \mathrm{In}$-PHMG (before and after radiolabeling, respectively). (c) Stability of ${ }^{111} \mathrm{In}$-PHMG in mouse serum (in vitro). 
to determine the serum stability of ${ }^{111} \mathrm{In}-\mathrm{PHMG}$. The retention time of free ${ }^{111} \mathrm{In}$ metal was less than $3 \mathrm{~min}$, which makes a large difference in the retention time than the ${ }^{111} \mathrm{In}-\mathrm{PHMG}$. The liberated free ${ }^{111} \mathrm{In}$ after $168 \mathrm{~h}$ incubation in the serum was as low as $12 \%$ and approximately $88 \%$ of ${ }^{111}$ In was retained with the parental molecule (Fig. 2(c)), which is enough stability for in vivo study.

\subsection{Biodistribution Studies in Sprague-Dawley Rats}

The biodistribution studies of ${ }^{111} \mathrm{In}$-PHMG were conducted in male SD rats. The radiolabeled PHMG was diluted in saline (aqueous $0.9 \% \mathrm{NaCl}$ solution) to a final concentration of $37 \mathrm{KBq} / 100 \mu \mathrm{L}$. The radiotracer was then administered to animals through three routes (intravenous injection, intratracheal instillation, and oral administration). The blood and nine organs were dissected and analyzed seven times, up to $168 \mathrm{~h}$ post-exposure, using $\gamma$-counter for quantitative measurement of PHMG in biological tissues. The radioactivity in excreta (urine and feces) was not collected in this experiment; thus, the biodistribution data would not represent all the activity administered.

The amount of PHMG distributed in tissues and blood is shown in Fig. 3 after decay correction of radioactivity at the end of intravenous injection $(\mathrm{t}=0 \mathrm{~h})$. The biodistribution data plotting the \%ID (Fig. 3(a)) exhibited the highest uptake in the liver (28.63\%ID at $0.5 \mathrm{~h}$ post-exposure) and the levels were increased over time. A similar trend was observed in the spleen, which represents the accumulation of PHMG in these organs. PHMG is highly distributed in the lungs (12.44\% $\mathrm{ID}$ at $0.5 \mathrm{~h}$ post-exposure), and the observed radioactivity decreased slowly within $168 \mathrm{~h}$. The uptake values in the lungs were to be more remarkable when the radioactivity was normalized to tissue mass (\%ID/g, Fig. 3(b)). At the initial time point, a noticeable uptake of ${ }^{111} \mathrm{In}-\mathrm{PHMG}$ was found in the lungs with $13.19 \% \mathrm{ID} / \mathrm{g}$, and this amount was higher than those of the liver $(5.31 \% \mathrm{ID} / \mathrm{g})$ and spleen $(3.99 \% \mathrm{ID} / \mathrm{g})$. In addition, only approximately $35 \%$ of the initial uptake value $(0.5 \mathrm{~h})$ in the lungs was reduced after $168 \mathrm{~h}$ post-injection ( $8.65 \% \mathrm{ID} / \mathrm{g})$. However, the radioactivity observed in the small and large intestines, and kidneys, which include excretory organs, were lower than in the liver and lungs. These results suggest high retention in the internal organs, as well as extremely slow clearance kinetics of intravenously injected PHMG.

In addition, we investigated the biodistribution after ${ }^{111} \mathrm{In}-\mathrm{PHMG}$ was exposed to SD rats through intratracheal instillation to observe the retention of PHMG in the lungs and the amount of translocation from the lungs. Radioactivity was mostly observed in the lungs at the initial time point $(0.5 \mathrm{~h}, 85.47 \% \mathrm{D} / \mathrm{g})$. The data demonstrated that administered PHMG was cleared slowly, with approximately $68 \%$ of radioactivity remaining in the lungs after $168 \mathrm{~h}$. Some PHMG was translocated to the extrapulmonary organs by passing the radiotracer through the air-blood barrier in the respiratory system [27, 28]. The quantification of radioactivity in some tissues
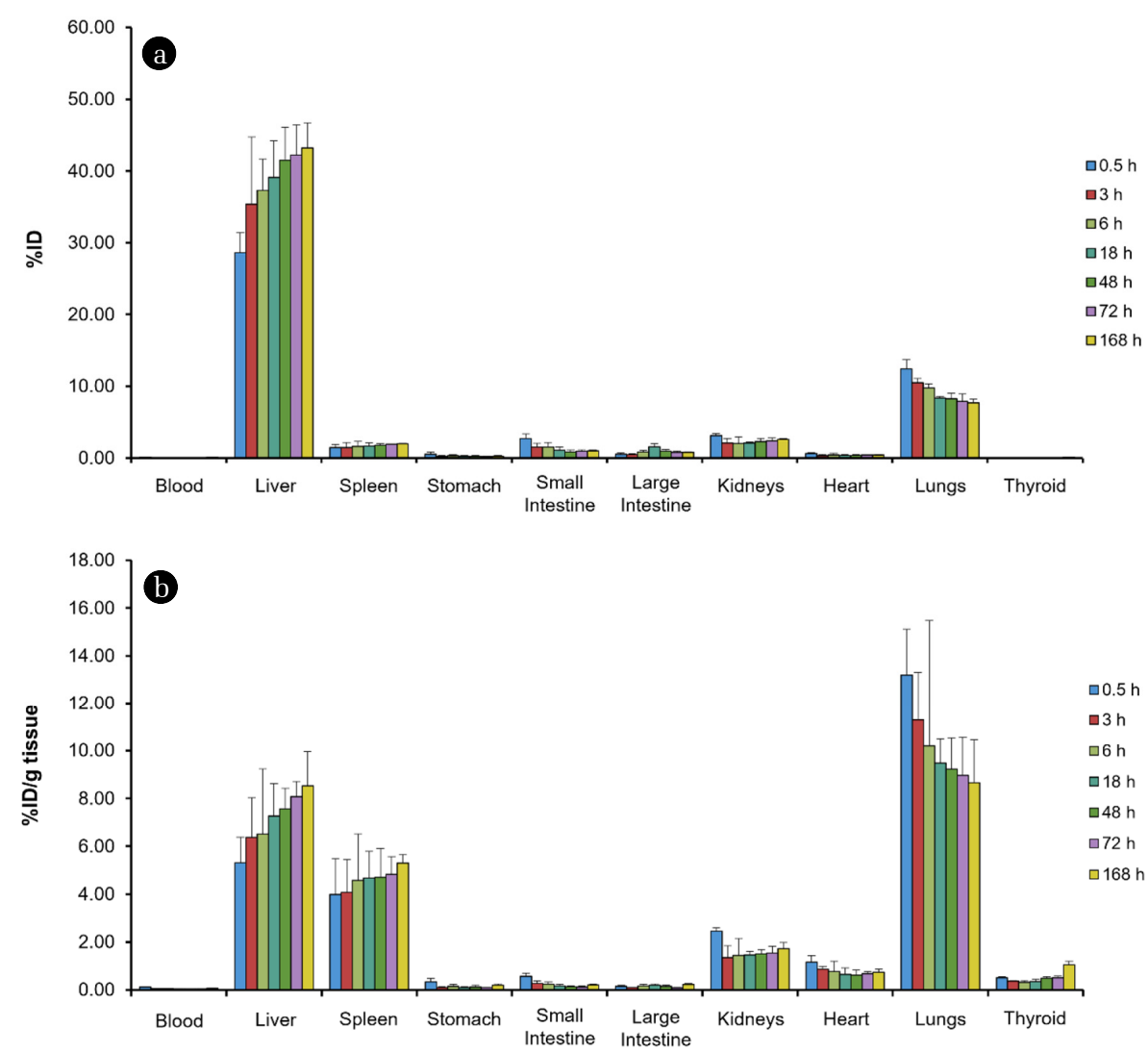

Fig. 3. Biological distribution of intravenously injected ${ }^{111}$ In-labeled polyhexamethylene guanidine $\left({ }^{111}\right.$ In-PHMG) expressed as (a) percentage of injected dose $(\% \mathrm{ID})$ and (b) \% ID/g of tissue, respectively. 
exhibited PHMG retention over time. For example, the uptake values of the liver were 1.12 and $1.74 \% \mathrm{ID} / \mathrm{g}$ at 0.5 and $168 \mathrm{~h}$ post exposure, respectively. In addition, an increase in uptake values was detected in the spleen. These trends were similar to those observed in Fig. 3, which exhibited persistent retention of PHMG in the lungs and liver, following intravenous administration. Taken together, prolonged exposure of PHMG through the respiratory system would have fatal effects to the lungs as well as other internal organs. The humidifier disinfectant can also be exposed to human via oral route. Interestingly, orally administered PHMG was cleared out in a day without significant translocation to other internal organs (i.e., lungs, heart, liver, and spleen) (Fig. 4(b)) from intestines. This result indicated that PHMG observed in gastrointestinal organs would have little effect on the uptake in the extraintestinal organs including lungs. The uptake values in each organ and the clearance rate could be related to the toxicity of PHMG. Our results in the present study are consistent with the recent toxicological studies, reporting that the oral lethal dose $\left(\mathrm{LD}_{50}\right)$ of PHMG for rats is 610 $\mathrm{mg} / \mathrm{kg}$ of body weight [29]. However, inhalation exposure is much more fatal, because the inhalation $\mathrm{LD}_{50}$ is observed to be $0.094-0.155$ $\mathrm{mg} / \mathrm{L}$ when it is exposed to rats for $4 \mathrm{~h}$.

Next, the biological distribution of PHMG was investigated through the molecular imaging method. Whole-animal SPECT images were obtained for $24 \mathrm{~h}$ post administration. For ${ }^{111} \mathrm{In}-\mathrm{PHMG}$ injected intravenously (Fig. 5(a)), a strong signal was detected in the internal organs, whereas the radiotracer exposed to the respira- tory tract showed localized accumulation in the lungs and the intensity of the signal was retained for 1 day (Fig. 5(b)), which is consistent with the biodistribution results. In contrast, the residual ${ }^{111}$ In-PHMG exposed using oral administration was restricted to the gastrointestinal tract, and the signal in the whole body decreased after $24 \mathrm{~h}$ exposure to the radiotracer [26].

Recent studies reveal the exposure of PHMG induced dose- and time-dependent cytotoxicity in various cells [9, 30-32]. The cationic guanidine groups in PHMG could bind negatively charged cellular components and biomolecules (e.g., phospholipids and proteins), which disrupted the membrane integrity, blocked the enzymatic pathway, and caused toxic effects on cell metabolism and survival [33, 34]. In addition, PHMG could consider interacting with intracellular organelles, which produce reactive oxygen species and lead to apoptosis [35]. According to the results in the present experiments, a part of intratracheally administered PHMG can be translocated to extrapulmonary organs. Moreover, the amount of PHMG distributed in some internal organs, such as the liver, spleen, and kidneys, did not significantly decrease for 1 week, which showed an unusual execration pattern. Considering that a single administration of radiolabeled PHMG in this study showed considerable accumulation of toxic chemicals, repeated exposure of PHMG should be responsible for severe toxicity in the respiratory system, as well as internal organs. The observed uptake values and SPECT imaging results would provide useful information to understand the toxicological effects of PHMG in various organs.
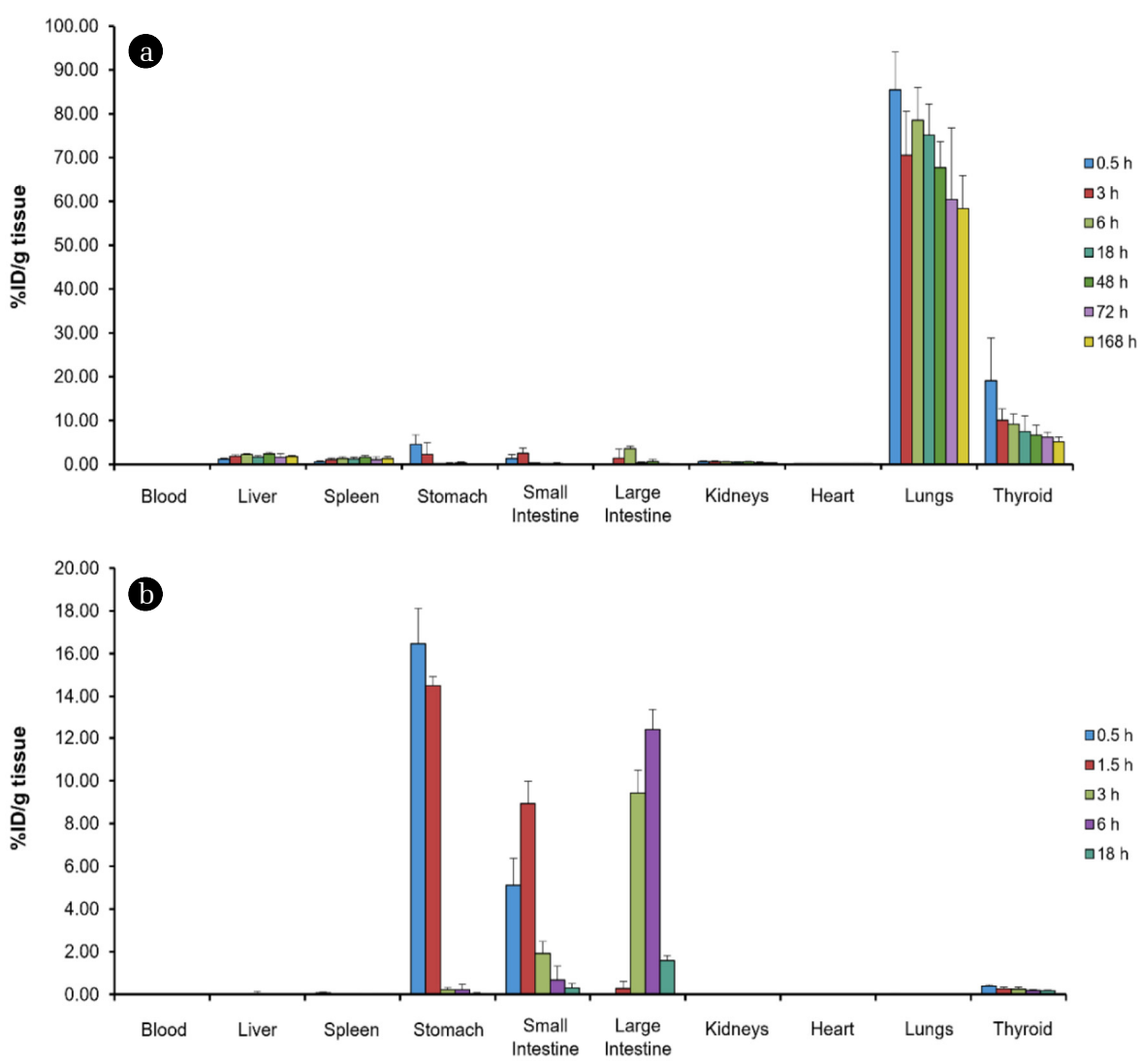

Fig. 4. Biological distribution of ${ }^{111} \mathrm{In}-\mathrm{PHMG}$ expressed as \% $\mathrm{ID} / \mathrm{g}$ of tissue; (a) intratracheal instillation and (b) oral administration. 


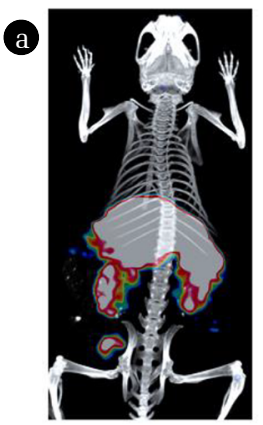

$0.5 \mathrm{~h}$

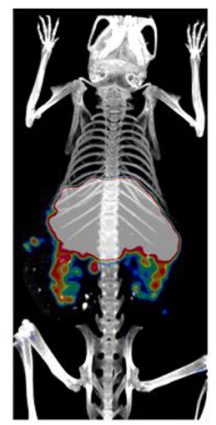

$3 \mathrm{~h}$

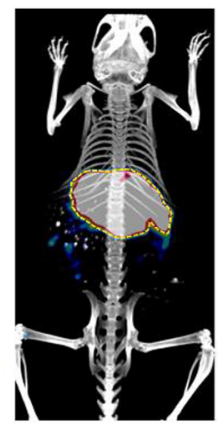

$18 \mathrm{~h}$

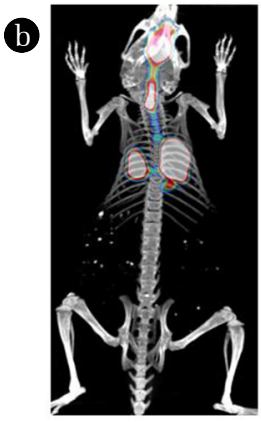

$0.5 \mathrm{~h}$

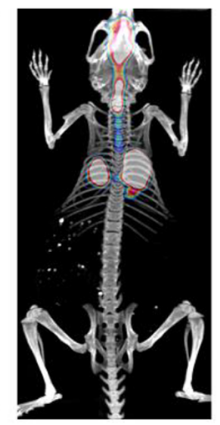

$3 \mathrm{~h}$

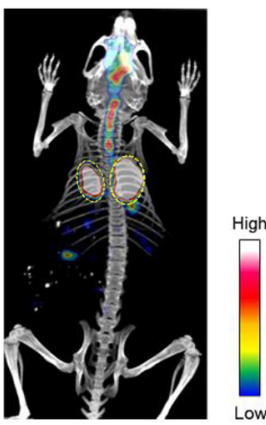

$24 \mathrm{~h}$

Fig. 5. Whole-body single-photon emission computed tomography/computed tomography images were obtained via (a) intravenous injection (yellow circle indicates the liver.) and (b) intratracheal instillation (yellow circle indicates the lungs.).

\section{Conclusions}

In this study, we demonstrate the radiolabeling of PHMG using ${ }^{111}$ In to measure its biological uptake in animal models. The radiotracer showed acceptable stability under the physiological conditions, and thus it was used to investigate the biological distribution of PHMG exposed to three administration routes. The results revealed that intravenously injected PHMG accumulated to a high level in the internal organs, suggesting that continual exposure to PHMG can be highly toxic to the human body. In addition, the data evidenced the transport of inhaled PHMG, which may be used to investigate the toxicological effects on extrapulmonary organs. The radioanalytical methods and molecular imaging strategy in this study can be used to investigate the risk assessment of other toxic polymers and disinfectants. Moreover, our approach can be used to assess the bioaccumulation and in vivo behavior of emerging contaminants, including inhaled fine particulate matter and ingested microplastics.

\section{Acknowledgment}

This work was supported by a research grant from Korea Food Research Institute (Grant number: E0210202-01) and also funded by Korea Ministry of Environment (MOE) as Environmental Health Action Program (Grant number: 2016001360012).

\section{Author Contributions}

S.M. (Assistant Professor), H.E.S. (Postdoctoral Researcher), and C.H.L. (Researcher) performed the experiments. H.S.S. (Principal Researcher) and S.-Y.L (Principal Researcher) contributed to the interpretation of work. J.E.P. (Graduate Student) analysed data and wrote the draft. J.J. (Assistant Professor) designed the experiments and wrote the manuscript.

\section{References}

1. Lachenmeier DW. Antiseptic Drugs and Disinfectants. In: Ray SD, ed. Side Effects of Drugs Annual. Amsterdam: Elsevier;
2015. p. 273-279.

2. Oulé MK, Quinn K, Dickman M, et al. Akwaton, polyhexamethylene-guanidine hydrochloride-based sporicidal disinfectant: A novel tool to fight bacterial spores and nosocomial infections. J. Med. Microbiol. 2012;61:1421-1427.

3. Vitt A, Sofrata A, Slizen V, et al. Antimicrobial activity of polyhexamethylene guanidine phosphate in comparison to chlorhexidine using the quantitative suspension method. Ann. Clin. Microbiol. Antimicrob. 2015;14:36.

4. Walczak M, Richert A, Burkowska-But A. The effect of polyhexamethylene guanidine hydrochloride (PHMG) derivatives introduced into polylactide (PLA) on the activity of bacterial enzymes. J. Ind. Microbiol. Biotechnol. 2014;41:1719-1724.

5. Lee E, Lee SY, Lee SJ. The past, present, and future of humidifier disinfectant-associated interstitial lung diseases in children. Clin. Exp. Pediatr. 2020;63:251-258.

6. Park JH, Kim HJ, Kwon GY, et al. Humidifier disinfectants are a cause of lung injury among adults in South Korea: A community-based case-control study. PLoS One. 2016;11: e0151849.

7. Song JA, Park HJ, Yang MJ, et al. Polyhexamethyleneguanidine phosphate induces severe lung inflammation, fibrosis, and thymic atrophy. Food Chem. Toxicol. 2014;69:267-275.

8. Park S, Lee K, Lee EJ, et al. Humidifier disinfectant-associated interstitial lung disease in an animal model induced by polyhexamethylene guanidine aerosol. Am. J. Respir. Crit. Care Med. 2014;190:706-708.

9. Song J, Jung KJ, Yoon SJ, Lee K, Kim B. Polyhexamethyleneguanidine phosphate induces cytotoxicity through disruption of membrane integrity. Toxicology 2019;414:35-44.

10. Kim HR, Lee K, Park CW, et al. Polyhexamethylene guanidine phosphate aerosol particles induce pulmonary inflammatory and fibrotic responses. Arch. Toxicol. 2016;90:617-632.

11. Kim MS, Kim SH, Jeon D, Kim HY, Lee K. Changes in expression of cytokines in polyhexamethylene guanidine-induced lung fibrosis in mice: Comparison of bleomycin-induced lung fibrosis. Toxicology 2018;393:185-192.

12. Park EJ, Park SJ, Kim S, Lee K, Chang J. Lung fibroblasts may play an important role in clearing apoptotic bodies of bronchial epithelial cells generated by exposure to PHMG-P-containing solution. Toxicol. Lett. 2018;286:108-119.

13. Song J, Jung KJ, Yang Mj, et al. Assessment of acute and repeated 
pulmonary toxicities of oligo(2-(2-ethoxy)ethoxyethyl guanidium chloride in mice. Toxicol. Res. 2021;37:99-113.

14. Kim SH, Kwon D, Lee S, et al. Polyhexamethyleneguanidine phosphate-induced cytotoxicity in liver cells is alleviated by tauroursodeoxycholic acid (TUDCA) via a reduction in endoplasmic reticulum stress. Cells 2019;8:1023.

15. Jung YS, Kim SH, Lee S, Son SW. Polyhexamethyleneguanidine phosphate-induced cytotoxicity is mediated by endoplasmic reticulum stress. FASEB J. 2020;34:1.

16. Lee J, Choi SJ, Jeong JS, et al. A humidifier disinfectant biocide, polyhexamethylene guanidine phosphate, inhalation exposure during pregnancy-induced toxicities in rats. J. Hazard. Mater. 2021;404:124007.

17. Lee YH, Seo DS. Toxicity of humidifier disinfectant polyhexamethylene guanidine hydrochloride by two-week whole body-inhalation exposure in rats. J. Toxicol. Pathol. 2020;33: 265-277.

18. Lee J, Choi SJ, Jeong JS, et al. Adverse postnatal developmental effects in offspring from humidifier disinfectant biocide inhaled pregnant rats. Chemosphere 2021;286:131636.

19. Jiang Y, Sun J, Xiong C, et al. Mass spectrometry imaging reveals in situ behaviors of multiple components in aerosol particles. Angew. Chem. Int. Ed. Engl. 2021;60(43):23225-23231.

20. Park K. An analysis of a humidifier disinfectant case from a toxicological perspective. Environ. Health Toxicol. 2016;31:e2016013.

21. Lee JY, Mushtaq S, Park JE, Shin HS, Lee SY, Jeon J. Radioanalytical techniques to quantitatively assess the biological uptake and in vivo behavior of hazardous substances. Molecules 2020;25:3985.

22. Lee CH, Shim HE, Song L, et al. Efficient and stable radiolabeling of polycyclic aromatic hydrocarbon assemblies: in vivo imaging of diesel exhaust particulates in mice. Chem. Commun. 2019;55:447-450.

23. Mushtaq S, Choi MH, Yang JE, et al. Technetium-99 m-based simple and convenient radiolabeling of Escherichia coli for in vivo tracking of microorganisms. J. Radioanal. Nucl. Chem. 2018;317:997-1003.

24. Welling MM, de Korne CM, Spa SJ, et al. Multimodal tracking of controlled Staphylococcus aureus infections in mice. ACS Infect. Dis. 2019;5:1160-1168.
25. Seo JW, Ingham ES, Mahakian L, et al. Positron emission tomography imaging of novel AAV capsids maps rapid brain accumulation. Nat. Commun. 2020;11:2102.

26. Shim HE, Lee JY, Lee CH, et al. Quantification of inhaled aerosol particles composed of toxic household disinfectant using radioanalytical method. Chemosphere 2018;207:649-654.

27. Buckley A, Warren J, Hodgson A, et al. Slow lung clearance and limited translocation of four sizes of inhaled iridium nanoparticles. Part. Fibre Toxicol. 2017;14:5.

28. Kreyling WG, Semmler-Behnke M, Takenaka S, Möller W. Differences in the biokinetics of inhaled nano- versus micrometer-sized particles. Acc. Chem. Res. 2013;46:714-722.

29. Lee HL, Byeon SH, Lee KS. Recommendation of occupational exposure limit through occupational hazard assessment of PHMG-phosphate. J. Korean Soc. Occup. Environ. Hyg. 2019;29: 13-20.

30. Jeong MH, Jeon MS, Kim GE, Kim HR. Polyhexamethylene guanidine phosphate induces apoptosis through endoplasmic reticulum sress in lung epithelial cells. Int. J. Mol. Sci. 2021;22:1215.

31. Paliienko KO, Veklich TO, Shatursky OY, et al. Membrane action of polyhexamethylene guanidine hydrochloride revealed on smooth muscle cells, nerve tissue, and rat blood platelets: A biocide driven pore-formation in phospholipid bilayers. Toxicol. In Vitro 2019;60:389-399.

32. Jung HN, Zerin T, Podder B, Song HY, Kim YS. Cytotoxicity and gene expression profiling of polyhexamethylene guanidine hydrochloride in human alveolar A549 cells. Toxicol. In Vitro 2014;28:684-692.

33. Kenzaoui BH, Vilà MR, Miquel JM, Cengelli F, Juillerat-Jeanneret L. Evaluation of uptake and transport of cationic and anionic ultrasmall iron oxide nanoparticles by human colon cells. Int. J. Nanomedicine 2012;7:1275-1286.

34. Cai J, Yue Y, Rui D, Zhang Y, Liu S, Wu C. Effect of chain length on cytotoxicity and endocytosis of cationic polymers. Macromolecules 2011;44:2050-2057.

35. Hunter AC, Moghimi SM. Cationic carriers of genetic material and cell death: A mitochondrial tale. Biochim. Biophys. Acta. 2010;1797:1203-1209. 